\title{
Compaction dynamics of crunchy granular material
}

\author{
François Guillard $^{1}{ }^{\star}$, Pouya Golshan ${ }^{2}$, Luming Shen ${ }^{1}$, Julio R. Valdès ${ }^{2}$, and Itai Einav ${ }^{1,3}$ \\ ${ }^{1}$ Particles and Grains Laboratory, School of Civil Engineering, The University of Sydney, Sydney, New South Wales 2006, Australia \\ ${ }^{2}$ Geo-Innovations Research Lab, Department of Civil, Construction, and Environmental Engineering, San Diego State University, \\ San Diego, California 92182, USA. \\ ${ }^{3}$ Department of Civil, Environmental \& Geomatic Engineering, Faculty of Engineering Science, University College London, London \\ WC1E 6BT, UK.
}

\begin{abstract}
Compaction of brittle porous material leads to a wide variety of densification patterns. Static compaction bands occurs naturally in rocks or bones, and have important consequences in industry for the manufacturing of powder tablets or metallic foams for example. Recently, oscillatory compaction bands have been observed in brittle porous media like snow or cereals. We will discuss the great variety of densification patterns arising during the compaction of puffed rice, including erratic compaction at low velocity, one or several travelling compaction bands at medium velocity and homogeneous compaction at larger velocity. The conditions of existence of each pattern are studied thanks to a numerical spring lattice model undergoing breakage and is mapped to the phase diagram of the patterns based on dimensionless characteristic quantities. This also allows to rationalise the evolution of the compaction behaviour during a single test. Finally, the localisation of compaction bands is linked to the strain rate sensitivity of the material.
\end{abstract}

When a brittle material undergoes compaction, a wide variety of compaction patterns can be observed. Static compaction bands are observed very commonly in nature, for example in bones, rocks such as sandstone [1-3], or in meteoritic impacts [4]. In industrial processes, compaction of brittle porous media is involved during fabrication of pharmaceutical drugs [5], or when using metallic foams [6] or honeycomb structures [7]. The localisation of the compaction in dense bands leads to inhomogeneities in the material, modifying its properties, which can have important consequences on its mechanical or chemical response. Usually, the bands of lower porosity resulting from the loading of material remain static in the medium. However, it has been shown recently that moving compaction bands can also appear during the compression of brittle porous media like puffed rice [8] or snow [9]. In these cases, the compaction zone travels within the medium, leading to oscillating compaction bands. However, the precise mechanism leading to the localisation of the compaction bands, and how this compression behaviour can be generalised to other type of brittle porous media remain elusive.

In this paper, we will first show that a wide variety of compaction patterns can appear when compressing a medium made of brittle porous material (puffed rice), including periodic travelling densification bands. These different compaction patterns will be explained using a simple spring lattice model [10]. The condition of appearance of the different patterns will be related to the characteristic

\footnotetext{
^e-mail: francois.guillard@sydney.edu.au
}
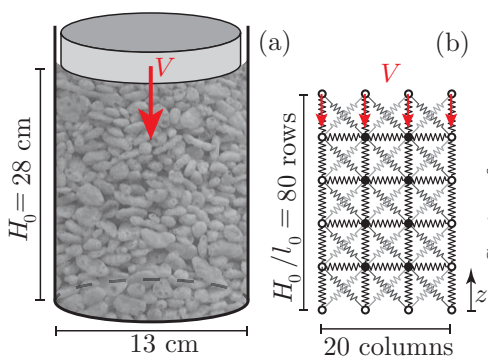

(c)

Figure 1. (a) Sketch of the experimental device for dry compaction of puffed rice at constant velocity. (b) Spring lattice geometry used in simulations. (c) Relationship between force and displacement for the simulated breakable springs.

times involved in the physical processes, and the evolution of the compaction behaviour over time will be discussed. Finally, the localisation of the compaction bands will be related to the strain rate sensitivity of the medium.

Fig. 1a shows the experimental device used for the compression of a dry brittle porous material. The medium is made of puffed rice placed in a transparent cylindrical tank up to a height $H_{0}$, compressed from the top at constant velocity $V$. The side view of the tank is recorded during the compaction, allowing us to measure the velocity field over time using Particle Image Velocimetry (PIV). Since the experiment has an axial symmetry, all the velocities are vertical and averaged horizontally, and the velocity depends only on the vertical position $z$ in the sam- 


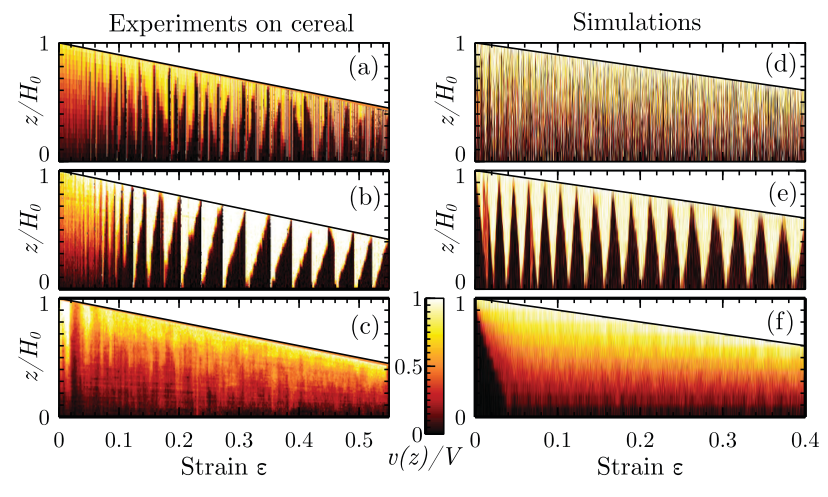

Figure 2. Heatmap of the medium velocity at a given depth $v$ scaled by top velocity $V$ as a function of scaled depth and strain, measured in experiments and simulations. (a,b,c) Experiments with $V=0.013 \mathrm{~mm} . \mathrm{s}^{-1}, 1.3 \mathrm{~mm} \cdot \mathrm{s}^{-1}$ and $300 \mathrm{~mm} \cdot \mathrm{s}^{-1}$,respectively. (d,e,f) Model results with $V=5 \times 10^{-6}, 2 \times 10^{-5}$ and $7 \times 10^{-5}$, respectively (with $k_{0}=2 \times 10^{-3}, F_{\mathrm{br}}^{0}=2 \times 10^{-6}, \eta=1 \times 10^{-3}$ ).

ple. Fig. 2a-c show the spatiotemporal velocity field for three different experiments with different compaction velocities. At low velocity, the compaction is mainly erratic, with different zones of the sample compacting at different time, without correlation between them. When the velocity is higher, the periodic compaction bands observed by Valdès et al. [8] are recovered. In that case the sample splits into two zones: a static zone at the bottom, and a zone moving at the velocity of the top compacting plate above. The boundary between these two zones moves upward over time. Finally, at the highest velocity, the whole sample compresses at the same time, leading to a homogeneous compaction, the velocity $v(z)$ at a given height $z$ being approximately given by $v(z)=\frac{V}{H} z$.

To better understand the origin of these various compaction patterns, numerical simulations are performed using a spring lattice model $[11,12]$. In this model, a lattice of nodes of mass $m$ is created, each of the node being linked to his neighbours by springs that are able to undergo repetitive breakage events (Fig. 1b). The lattice is completely regular, and all the springs have same breakage law and parameters, so there is no disorder in the simulations. The equation of motion are solved for each of the nodes using a verlet algorithm with timestep $\Delta t$ and the lattice is deformed accordingly. In addition to the force due to the springs, each node is also submitted to a global viscous damping force $\eta v_{j}$, where $\eta$ is the viscosity and $v_{j}$ the velocity of node $j$. The springs in the lattice are able to break multiple time when they are compressed, following the force-displacement law of Fig. 1c. When the force in a spring overtake its strength $F_{\text {br }}$, it breaks, ie. its equilibrium length $l$ is set to its current length and its stiffness $k$ and strength $F_{\mathrm{br}}$ are increased. If $i$ denotes the number of time a given spring already broke, the increase of stiffness and strength is set as $k_{i+1}=k_{i}+a k_{0}$ and $F_{\mathrm{br}}^{i+1}=F_{\mathrm{br}}^{i}+a F_{\mathrm{br}}^{0}$, where $a=0.01$. During compaction of the lattice, each spring is therefore able to break as many time as needed, each breakage event releasing the force in the corresponding spring. The simulations are non-dimensional, with

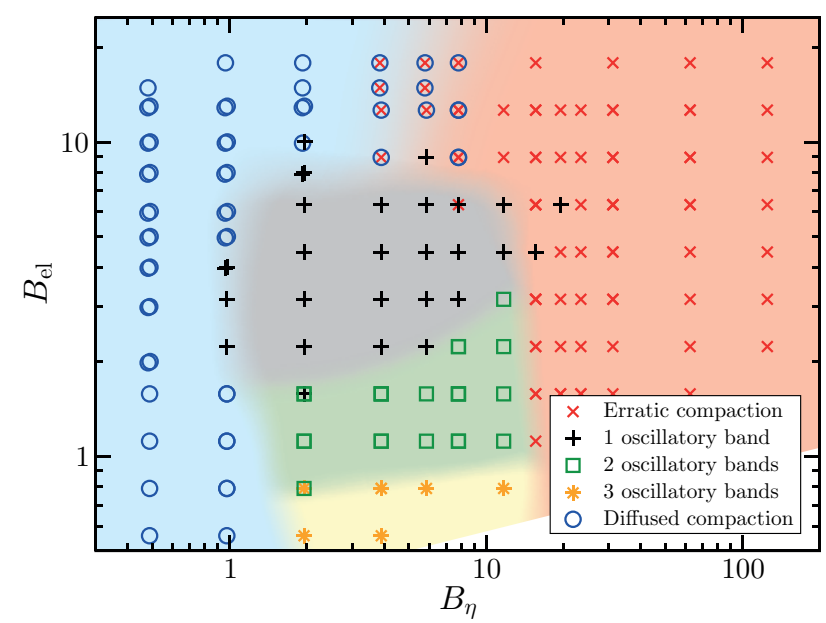

Figure 3. Compaction patterns observed in model simulations as a function of the dimensionless numbers $B_{\eta}$ and $B_{\mathrm{el}}$.

basic units of mass $[M]=m$, length $[L]=l_{0}$ and time $[T]=\Delta t$. Velocity field are coarse-grained from the node velocity [13].

When performing these simulations with different compaction velocity, compaction patterns similar to the ones appearing in experiment are observed. Fig. 2d-f shows that we recover the erratic compaction, single travelling compaction band and homogenous compaction observed in the experimental data (Fig. 2a-c).

Interestingly other compaction patterns are also observed in the simulations, corresponding to multiple compaction bands travelling through the sample at the same time. In fact, up to three oscillatory bands have been observed in the spring lattice model simulations. It remains unclear if these pattern also occur in the experiment as they may be too faint to be observed.

The model allows us to test the effect of the physical parameters on the observed compaction patterns. Three different characteristic times can be extracted from the simulation. The elastic time $\tau_{\mathrm{el}}$ corresponds to the time needed for an elastic wave to travel the height of the sample. The viscous time $\tau_{\eta}$ corresponds to the slow down time of a node due to viscosity. Finally, the breakage time corresponds to the typical time between two successive breakage of a node given the strain rate that is applied. These times are defined from the simulation parameters with:

$$
\tau_{\mathrm{br}}=\frac{F_{\mathrm{br}}}{k l} \frac{H}{V} ; \quad \tau_{\mathrm{br}}=\frac{H}{l} \sqrt{\frac{m}{k}} ; \quad \tau_{\eta}=\frac{m}{\eta}
$$

where the indices $i$ have been removed for simplicity. Note that in principle these characteristic times evolve during a given simulation, so we start by focusing only on their initial value and on the first identifiable compaction pattern on a given test.

From these characteristic times two dimensionless groups can be derived, an 'elasto-breakage' number $B_{\mathrm{el}}$ 
and a 'visco-breakage' number $B_{\eta}$, defined as:

$$
B_{\mathrm{el}}=\frac{\tau_{\mathrm{br}}}{\tau_{\mathrm{el}}}=\frac{F_{\mathrm{br}}}{V \sqrt{k m}} ; B_{\eta}=\frac{\tau_{\mathrm{br}}}{\tau_{\eta}}=\frac{F_{\mathrm{br}} H \eta}{k l V m}
$$

These two dimensionless numbers allow to clarify the range of parameters leading to the observed compaction patterns. Fig. 3 shows the patterns observed as a function of $B_{\mathrm{el}}$ and $B_{\eta}$. At high $B_{\eta}$, when the dissipation is high, the compaction is erratic since when breakage happens in the medium the elastic energy is rapidly dissipated and therefore there is no correlation between the breakage event in time. At low $B_{\eta}$ on the contrary, there is very low dissipation and the medium is very weak so the whole sample breaks at the same time, leading to a homogeneous compaction. In between these two extremes, single of multiple oscillatory compaction band can appear, depending on $B_{\mathrm{el}}$. Interestingly, the range of parameter leading to these bands gets smaller as the number of bands increases, similar to what is observed in the physics of bifurcation near phase transition. This limited range of parameters could also explain that these multiple oscillatory compaction bands have not been observed experimentally.

To see how general these results are for different type of materials, we also performed simulations where the dissipation mechanism is changed from global viscosity at the nodes to local viscosity inside each bond (Kelvin-Voigt model). The force in the spring is therefore not only computed from their extension, but also from the relative velocity of the nodes $i$ and $j$ it is attached to, adding a force $\eta\left(\mathbf{v}_{j}-\mathbf{v}_{i}\right)$ on node $i$. The characteristic viscous time is therefore modified to $\tau_{\text {eta }}^{m}=\eta / k$ to account for the change in the damping mechanism, and the 'visco-breakage' number to $B_{\eta}^{m}=\tau_{\mathrm{br}} / \tau_{\eta}^{m}$. Fig. 4 shows the phase diagram for this model. Clearly the patterns observed in this case are very similar to the one observed with global damping, and the phase diagram is only marginally modified. Travelling compaction bands seems to be independent of the details of the breakage and dissipation mechanism, and therefore should be a general behaviour appearing in the compaction of brittle porous media for a certain range of parameters, which is also supported by their observation in snow [9] as well as puffed rice.

As mentioned earlier, the previous phase diagrams are based on the first compaction pattern observed in the simulations. However, $B_{\mathrm{el}}$ and $B_{\eta}^{m}$ are evolving during a given simulation, due to the decrease of the height of the sample and the increase of the spring breakage inside the sample that modifies the average strength and stiffness of the springs. By taking these effects into account, one can rewrite $B_{\mathrm{el}}$ and $B_{\eta}^{m}$ over time, with $\bar{l}$ the average number of breakage per spring, and $t$ the simulation time:

$$
B_{\mathrm{el}}=\frac{F_{0} \sqrt{1+\bar{\imath} a}}{V} \sqrt{\frac{m}{k_{0}}} ; B_{\eta}^{m}=\frac{F_{0}(1+\bar{\imath} a)\left(H_{0}-V t\right)}{V \eta l} .
$$

Interestingly, the evolution of $B_{\mathrm{el}}$ and $B_{\eta}^{m}$ over time implies that the same sample can experience different patterns of compaction over one simulation. Fig. 5 shows one particular simulation with bond viscosity where the material

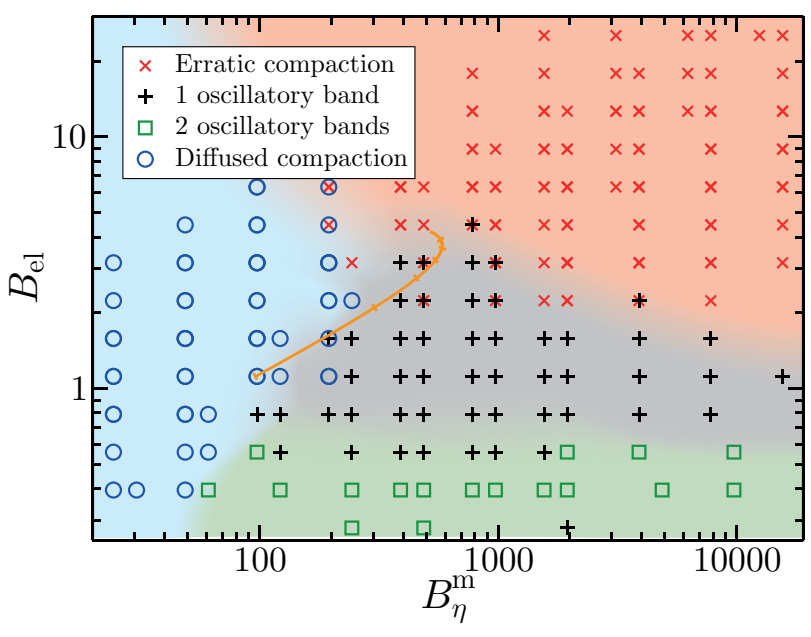

Figure 4. Compaction patterns observed in model simulations as a function of the dimensionless numbers $B_{\eta}^{m}$ and $B_{\mathrm{el}}$ for bond viscosity. The continuous line is the path followed by the simulation presented in Fig. 5, the ticks on the line are equally spaced in time.

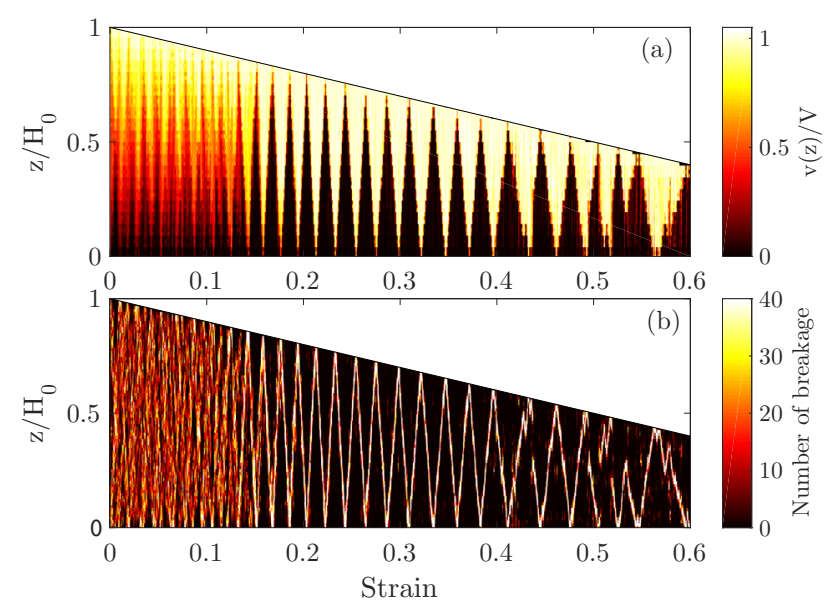

Figure 5. Velocity field (a) and total breakage events per row and per timestep (b) function of the strain for a given simulation. The simulation follows the path in the phase space indicated in figure 4. $V=2 \times 10^{-5}, \eta=0.02, k_{0}=5 \times 10^{-4}, F_{\mathrm{br}}^{0}=5 \times 10^{-7}$.

moves along the orange line in Fig. 4 during the experiments. The simulation starts with a diffuse compaction patterns. As the material breaks, both $B_{\mathrm{el}}$ and $B_{\eta}^{m}$ increase, and the sample reaches the region where a travelling compaction band can localise and propagate into the medium. Toward the end of the simulation, the breakage rate decreases on average because the bonds that were initially inclined at $45^{\circ}$ are now almost horizontal flat, and therefore the height dependency term in $B_{\eta}^{m}$ takes over and reduces its value, while $B_{\mathrm{el}}$ keeps increasing slowly. The sample then starts to display more erratic compaction behaviour as it reaches this configuration. The same simulation can lead to multiple different compaction patterns over time because of the evolution of the geometry of the sample and of the internal bond properties due to breakage. 


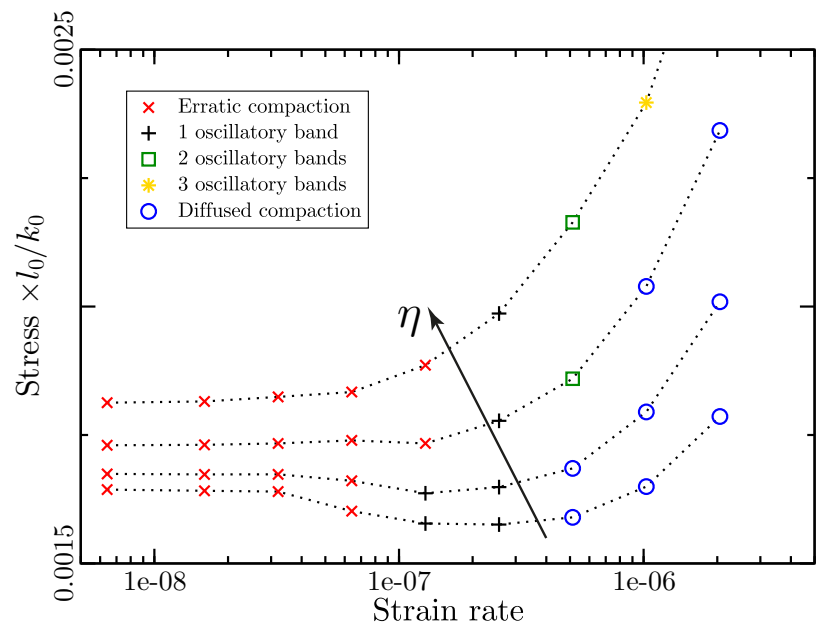

Figure 6. Stress as a function of compaction strain-rate for different simulations with global viscosity. Points on the same dotted line have the same viscosity, respectively $\eta=2.5 \times 10^{-4}$, $\eta=5 \times 10^{-4}, \eta=1 \times 10^{-3}, \eta=2 \times 10^{-3} . k_{0}=4 \times 10^{-3}$, $F_{\text {br }}^{0}=4 \times 10^{-6}$.

The phenomenon of localisation of compaction bands is reminiscent of similar behaviour observed in the extension of metallic alloys [14-16]. In this case, applying a tensile load to the material results in intermittent stress drops or 'jerky flow'. This instability is known to appear because of the strain-rate softening properties of the material, ie. the softening of the material with increasing pulling velocity, that leads to the instability of some strain rate states. It is relatively simple to check if the same kind of behaviour is observed in our model. To this end, the applied stress at the top is measured in simulation for different compacting velocity along with the identified compaction pattern (Fig. 6). It is clear that the oscillatory compaction bands are associated with the strain-rate softening of the material, at least when the dissipation is low. Interestingly, when $\eta$ is increased, travelling compaction bands can still be observed for similar strain-rate range even if the medium does not seem to show macroscopic strain-rate softening. This is due to the fact that only bond level strain-rate softening is required to have localisation of a compaction band, whereas the bulk material can actually exhibit strain-rate hardening as a whole. This effect can have important consequences since its suggests that assessing the mechanical properties of a material at the macroscopic level may hide some important microscopic properties that can greatly modify the behaviour of the material.

In conclusion, a wide variety of compaction behaviour have been observed when brittle porous materials are compressed. In absence of water, when the grains breaks due to the applied stress only, compaction leads to either erratic behaviour at low velocity, single or multiple compaction bands oscillating in the medium when the velocity is increased, and homogeneous compaction at large velocity. These patterns have been observed experimentally and modelled numerically using a lattice of springs able to undergo multiple breakage events. The generality of the processes involved in the apparition of these different behaviours suggest that other brittle porous material should exhibit similar phenomena. This effect of localisation of compaction bands is related to the shear-rate sensitivity of the material at microscopic level, and can be linked to similar stress localisation effects observed in metallic alloys [16] and to the apparition of cnoidal or solitary wave in non-linear dynamic systems [17].

\section{Acknowledgements}

I.E. and L.S. thank the Australian Research Council for support through project DP130101291. J.R.V. thanks the USA National Science Foundation for support through Grant CBET1336952.

\section{References}

[1] W.A. Olsson, Mechanics of Materials 33, 659 (2001)

[2] V. Vajdova, T.F. Wong, Geophysical Research Letters 30, 1 (2003)

[3] P. Baud, E. Klein, T.F. Wong, Journal of Structural Geology 26, 603 (2004)

[4] S.G. Love, F. Hörz, D.E. Brownlee, Icarus 105, 216 (1993)

[5] C.Y. Wu, O.M. Ruddy, A.C. Bentham, B.C. Hancock, S.M. Best, J.A. Elliott, Powder Technology 152, 107 (2005)

[6] A.F. Bastawros, H. Bart-Smith, A.G. Evans, Journal of the Mechanics and Physics of Solids 48, 301 (1999)

[7] S. Papka, S. Kyriakides, Acta Materialia 46, 2765 (1998)

[8] J.R. Valdes, F.L. Fernandes, I. Einav, Granular Matter 14, 71 (2011)

[9] T.W. Barraclough, J.R. Blackford, S. Liebenstein, S. Sandfeld, T.J. Stratford, G. Weinländer, M. Zaiser, arXiv:1501.0218 (2015), arXiv: 1501.0218

[10] F. Guillard, P. Golshan, L. Shen, J.R. Valdes, I. Einav, Nature Physics 11, 835 (2015)

[11] R. Katsman, E. Aharonov, H. Scher, Mechanics of Materials 37, 143 (2005)

[12] S. Kale, M. Ostoja-starzewski, Phys. Rev. E 042405 , 1 (2014)

[13] B.J. Glasser, I. Goldhirsch, Physics of Fluids 13, 407 (2001)

[14] P. Penning, Acta Metallurgica 20, 1169 (1972)

[15] L.P. Kubin, Y. Estrin, Acta Metallurgica 33, 397 (1985)

[16] T.A. Lebedkina, M.A. Lebyodkin, Acta Materialia 56, 5567 (2008)

[17] E. Veveakis, K. Regenauer-Lieb, Journal of the Mechanics and Physics of Solids 78, 231 (2015) 